Internat. J. Math. \& Math. Sci.

Vol. 24, No. 12 (2000) 793-806

S0161171200020068

(c) Hindawi Publishing Corp.

\title{
SOME DETAILS OF PROOFS OF THEOREMS RELATED TO THE QUANTUM DYNAMICAL YANG-BAXTER EQUATION
}

\author{
TOM H. KOORNWINDER
}

(Received 7 July 2000)

\begin{abstract}
This paper of tutorial nature gives some further details of proofs of some theorems related to the quantum dynamical Yang-Baxter equation. This mainly expands proofs given in "Lectures on the dynamical Yang-Baxter equation" by Etingof and Schiffmann, math.QA/9908064. This concerns the intertwining operator, the fusion matrix, the exchange matrix and the difference operators. The last part expands proofs given in "Traces of intertwiners for quantum groups and difference equations, I" by Etingof and Varchenko, math.QA/9907181. This concerns the dual MacdonaldRuijsenaars equations.
\end{abstract}

Keywords and phrases. Quantum dynamical Yang-Baxter equation, intertwining operator, fusion matrix, exchange matrix, weighted trace function, dual Macdonald-Ruijsenaars equations.

2000 Mathematics Subject Classification. Primary 17B37.

1. Introduction. The quantum dynamical Yang-Baxter equation (QDYBE) was first considered by Gervais and Neveu [9], with motivation from physics (for monodromy matrices in Liouville theory). A general form of QDYBE with spectral parameter was presented by Felder [7, 8] at two major congresses. The corresponding classical dynamical Yang-Baxter equation (CDYBE) was presented there as well. Next Etingof and Varchenko started a program to give geometric interpretations of solutions of CDYBE [3] and of QDYBE [4] in the case without spectral parameter. In the context of this program they pointed out a method to obtain solutions of QDYBE by the so-called exchange construction [5]. This uses, for any simple Lie algebra $\mathfrak{g}$, representation theory of $U(\mathfrak{g})$ or of its quantized version $U_{q}(\mathfrak{g})$ in order to define successively the intertwining operator, the fusion matrix and the exchange matrix. The matrix elements of the intertwining operator and of the exchange matrix generalize respectively the Clebsch-Gordan coefficients and the Racah coefficients to the case where the first tensor factor is a Verma module rather than a finite dimensional irreducible module. The exchange matrix is shown to satisfy QDYBE. Etingof and Varchenko also started in [6] a related program to connect the above objects with weighted trace functions and with solutions of the ( $q$-)Knizhnik-Zamolodchikov-Bernard equation (KZB or $q$ KZB).

A nice introduction to the topics indicated above was recently given by Etingof and Schiffmann [2]. While I was reading this paper in connection with a seminar in Amsterdam during the fall of 1999, I added some details of proofs for my own convenience, and I put these notes in $\mathrm{TEX}_{\mathrm{E}}$ in order that the other participants in the seminar could 
take profit of it. I put these informal notes on my homepage. Since Version 2 of [2] is now referring to these notes, I decided to give my notes a more official status.

I want to emphasize that these notes are purely meant as a tutorial giving some details of the sometimes rather succinct proofs in [2]. However, I did not try to cover the full contents of [2]. Most of my paper only treats the $q=1$ case. Only the second part of the section on the exchange matrix also covers the quantum case. In general, the extension to the quantum case will usually be straightforward.

As for the contents, Sections 2, 3, and 4, respectively deal with the intertwining operator, the fusion matrix and the exchange matrix. In [2] these topics are all covered in Section 2. Sections 5 and 6 on difference operators and on weighted trace functions address some topics in Section 9 of [2] (Transfer matrices and generalized MacdonaldRuijsenaars equations). The details of proofs in Section 6 concern $q=1$ analogues of proofs given in Section 3 of [6] in connection with the dual Macdonald-Ruijsenaars equations.

I want to call attention to one conceptual aspect. This concerns formulas (4.11) and (4.12). The first formula expresses an exchange matrix $R_{U, V \otimes W}(\lambda)$ after shifted conjugation by the fusion matrix $J_{V W}(\lambda)$ as a product of $R_{U V}(\lambda)$ (with appropriately shifted $\lambda$ ) and $R_{U W}(\lambda)$. The second formula is analogous. These formulas are not explicitly given in [2], but they do occur in [6] without getting particular emphasis. They can be used in order to prove that $R(\lambda)$ satisfies QDYBE. This is analogous to the role of the quasi-triangularity property of the (non-dynamical) universal $R$-matrix for proving the QYBE in that case. In fact, it is possible to see (4.11) and (4.12) in the context of a certain quasitriangular quasi-Hopf algebra, see Babelon, Bernard, and Billey [1, Section 3]) for the quantum sl(2) case.

Notation. Throughout this paper I denote by [E-S] the paper [2] by Etingof and Schiffmann, and by [E-V] the paper [6] by Etingof and Varchenko.

2. The intertwining operator. First I make two preliminary remarks in preparation of the proof of [E-S], Proposition 2.2.

Let $\mathfrak{g}$ be a Lie algebra with Lie subalgebra $\uparrow$, and let $V$ be a $\uparrow$-module. Then

$$
\operatorname{Ind}_{\mathfrak{\Upsilon}}^{\mathfrak{g}} V:=U(\mathfrak{g}) \otimes_{\mathbb{\Gamma}} V \quad \text { with } a \cdot\left(u \otimes_{\mathfrak{\Gamma}} v\right):=(a u) \otimes_{\mathfrak{\Upsilon}} v(a \in \mathfrak{g}, u \in U(\mathfrak{g}), v \in V) .
$$

Let $W$ be a $\mathfrak{g}$-module. Then Frobenius reciprocity states that there is an isomorphism of linear spaces

$$
f \longleftrightarrow F: \operatorname{Hom}_{\uparrow}(V, W) \longleftrightarrow \operatorname{Hom}_{\mathfrak{g}}\left(U(\mathfrak{g}) \otimes_{\mathfrak{\Gamma}} V, W\right)
$$

given by $F\left(u \otimes_{\uparrow} v\right):=u \cdot f(v), f(v):=F\left(1 \otimes_{\uparrow} v\right)(u \in U(\mathfrak{g}), v \in V)$.

For the other remark let $\mathfrak{g}$ be a Lie algebra and let $Z, W, V$ be $\mathfrak{g}$-modules. Then there is an isomorphism of linear spaces

$$
f \longleftrightarrow F: \operatorname{Hom}_{\mathfrak{g}}(Z, W \otimes V) \longleftrightarrow \operatorname{Hom}_{\mathfrak{g}}\left(Z \otimes W^{*}, V\right)
$$

given by $F\left(z \otimes w^{*}\right)=\left\langle f(z), w^{*}\right\rangle\left(z \in Z, w^{*} \in W^{*}\right)$. 
Proof of [E-S], Proposition 2.2. We have a composition of five isomorphisms

$$
\begin{aligned}
\Phi & \longleftrightarrow \Phi_{1} \longleftrightarrow \Phi_{2} \longleftrightarrow \Phi_{3} \longleftrightarrow \Phi_{4} \longleftrightarrow \Phi_{5}=\langle\Phi\rangle: \operatorname{Hom}_{\mathfrak{g}}\left(U(\mathfrak{g}) \otimes_{\mathfrak{h} \otimes \mathfrak{n}_{+}} \mathbb{C}_{\lambda}, M_{\mu} \otimes V\right) \\
& \longleftrightarrow \operatorname{Hom}_{\mathfrak{h} \otimes \mathfrak{n}_{+}}\left(\mathbb{C}_{\lambda}, M_{\mu} \otimes V\right) \longleftrightarrow \operatorname{Hom}_{\mathfrak{h} \otimes \mathfrak{n}_{+}}\left(\mathbb{C}_{\lambda} \otimes M_{\mu}^{*}, V\right) \\
& \longleftrightarrow \operatorname{Hom}_{\mathfrak{h} \otimes \mathfrak{n}_{+}}\left(U\left(\mathfrak{n}_{+}\right) \otimes_{\mathfrak{h}} \mathbb{C}_{-\mu}, V \otimes \mathbb{C}_{\lambda}^{*}\right) \longleftrightarrow \operatorname{Hom}_{\mathfrak{h}}\left(\mathbb{C}_{-\mu}, V \otimes \mathbb{C}_{\lambda}^{*}\right) \\
& \longleftrightarrow \operatorname{Hom}_{\mathfrak{h}}\left(\mathbb{C}_{\lambda} \otimes \mathbb{C}_{-\mu}, V\right),
\end{aligned}
$$

where

$$
\begin{aligned}
& \Phi_{1}\left(x_{\lambda}\right):=\Phi\left(x_{\lambda}\right), \\
& \Phi_{2}\left(x_{\lambda} \otimes u^{*}\right):=\left\langle\Phi\left(x_{\lambda}\right), u^{*}\right\rangle \quad\left(u^{*} \in M_{\mu}^{*}\right), \\
& \Phi_{3}\left(u^{*}\right):=\left\langle\Phi\left(x_{\lambda}\right), u^{*}\right\rangle \otimes x_{\lambda}^{*} \quad\left(u^{*} \in M_{\mu}^{*} \simeq \mathbb{C}_{-\mu} \otimes_{\mathfrak{h}} U\left(\mathfrak{n}_{+}\right)\right), \\
& \Phi_{4}\left(x_{-\mu}\right):=\left\langle\Phi\left(x_{\lambda}\right), x_{\mu}^{*}\right\rangle \otimes x_{\lambda}, \\
& \Phi_{5}\left(x_{\lambda} \otimes x_{-\mu}\right):=\left\langle\Phi\left(x_{\lambda}\right), x_{\mu}^{*}\right\rangle=\langle\Phi\rangle .
\end{aligned}
$$

PROOF THAT THE COEFFICIENTS OF $\Phi_{\lambda}^{v}$ ARE RATIONAL IN $\lambda$ (STATEMENT IN PARAGRAPH AFTER THE PROOF OF [E-S], PROPOSITION 2.2; THE PROOF BELOW IS ESSENTIALLY DUE TO ERIC OPDAM). Let $\alpha_{1}, \ldots, \alpha_{N}$ be the positive roots (the elements of $\Delta^{+}$). Let $V$ be a finite-dimensional $\mathfrak{g}$-module, and let $v \in V \backslash\{0\}$ be $\mathfrak{h}$-homogeneous. Consider the Verma module $M_{\lambda-w t(v)}$ for generic values of $\lambda \in \mathfrak{h}^{*}$, where it is irreducible. By Proposition 2.2, there is a unique $\mathfrak{g}$-intertwining linear map $\Phi_{\lambda}^{v}: M_{\lambda} \rightarrow$ $M_{\lambda-\mathrm{wt}(v)} \otimes V$ such that

$$
\Phi_{\lambda}^{v}\left(x_{\lambda}\right)=\sum_{k_{1}, \ldots, k_{N} \geq 0} f_{\alpha_{1}}^{k_{1}} \cdots f_{\alpha_{N}}^{k_{N}} \cdot x_{\mu} \otimes v_{k_{1}, \ldots, k_{N}} \quad \text { with } v_{0, \ldots, 0}=v .
$$

Here $\mu:=\lambda-\operatorname{wt}(v)$. Clearly wt $\left(v_{k_{1}, \ldots, k_{N}}\right)=\lambda-\mu+k_{1} \alpha_{1}+\cdots+k_{N} \alpha_{N}$. It is sufficient to show that the $v_{k_{1}, \ldots, k_{N}}$ are rational in $\lambda$.

The unique existence of $\Phi_{\lambda}^{v}$ satisfying the above conditions is equivalent to the unique existence of $w \in M_{\mu} \otimes V \operatorname{such}$ that $\operatorname{wt}(w)=\lambda, e_{\alpha_{i}} \cdot w=0$ for $i=1, \ldots, N$ and such that $w$ has the form of the right-hand side of (2.6) with $v_{0, \ldots, 0}=v$. We show that the unique existence of $w$ with these properties implies that the $v_{k_{1}, \ldots, k_{N}}$ are rational in $\lambda$.

Note that

$$
e_{\alpha_{i}} f_{\alpha_{1}}^{k_{1}} \cdots f_{\alpha_{N}}^{k_{N}} \cdot x_{\mu}=\sum_{\begin{array}{c}
l_{1}, \ldots, l_{N} \geq 0, \\
k_{1} \alpha_{1}+\cdots+k_{N} \alpha_{N}= \\
\alpha_{i}+l_{1} \alpha_{1}+\cdots+l_{N} \alpha_{N}
\end{array}} p_{i ; l_{1}, \ldots, l_{N}}^{k_{1}, \ldots, k_{N}}(\lambda) f_{\alpha_{1}}^{l_{1}} \cdots f_{\alpha_{N}}^{l_{N}} \cdot x_{\mu}
$$

with $p_{i ; l_{1}, \ldots, l_{N}}^{k_{1}, \ldots, k_{N}}(\lambda)$ polynomial in $\lambda$. So, for $i=1, \ldots, N$, we have

$$
0=e_{\alpha_{i}} \cdot w=\sum_{l_{1}, \ldots, l_{N}} f_{\alpha_{1}}^{l_{1}} \cdots f_{\alpha_{N}}^{l_{N}} \cdot x_{\mu} \otimes\left(e_{\alpha_{i}} \cdot v_{l_{1}, \ldots, v_{N}+} \sum_{\begin{array}{c}
k_{1}, \ldots, k_{N} \geq 0, \\
k_{1} \alpha_{1}+\ldots+k_{N} \alpha_{N}= \\
\alpha_{i}+l_{1} \alpha_{1}+\cdots+l_{N} \alpha_{N}
\end{array}} p_{i ; l_{1}, \ldots, l_{N}}^{k_{1}, \ldots, k_{N}}(\lambda) v_{k_{1}, \ldots, k_{N}}\right) .
$$


So the inhomogeneous system of linear equations in the coordinates of the vectors $v_{l_{1}, \ldots, l_{N}}\left(l_{1}, \ldots, l_{N}\right.$ nonnegative integers, not all 0$)$ given by

$$
e_{\alpha_{i}} \cdot v_{l_{1}, \ldots, v_{N}}+\sum_{\begin{array}{c}
k_{1}, \ldots, k_{N} \geq 0, \\
k_{1} \alpha_{1}+\cdots+k_{N} \alpha_{N}= \\
\alpha_{i}+l_{1} \alpha_{1}+\cdots+l_{N} \alpha_{N}
\end{array}} p_{i ; l_{1}, \ldots, l_{N}}^{k_{1}, \ldots, k_{N}}(\lambda) v_{k_{1}, \ldots, k_{N}}=0 \quad(i=1, \ldots, N)
$$

has, for generic $\lambda$, a unique solution. Since the coefficients are polynomials in $\lambda$ it follows that the solution must be rational in $\lambda$.

\section{The fusion matrix}

Proof of [E-S], Proposition 2.3, PART 2.

$$
\Phi_{\lambda}^{v}\left(x_{\lambda}\right) \in x_{\lambda-\mathrm{wt}(v)} \otimes v+M_{\lambda-\mathrm{wt}(v)}[<\lambda-\mathrm{wt}(v)] \otimes V[>\mathrm{wt}(v)] .
$$

Hence

$$
\Phi_{\lambda}^{v}\left(M_{\lambda}[<\lambda]\right) \subset x_{\lambda-\mathrm{wt}(v)} \otimes V[<\mathrm{wt}(v)]+M_{\lambda-\mathrm{wt}(v)}[<\lambda-\mathrm{wt}(v)] \otimes V .
$$

It follows that

$$
\begin{aligned}
& \left(\Phi_{\lambda-\operatorname{wt}(v)}^{w} \otimes 1\right)\left(\Phi_{\lambda}^{v}\left(x_{\lambda}\right)\right) \\
& \in \Phi_{\lambda-\operatorname{wt}(v)}^{w}\left(x_{\lambda-\operatorname{wt}(v)}\right) \otimes v+\Phi_{\lambda-\operatorname{wt}(v)}^{w}\left(M_{\lambda-\operatorname{wt}(v)}[<\lambda-\operatorname{wt}(v)]\right) \otimes V[>\operatorname{wt}(v)] \\
& \subset x_{\lambda-\operatorname{wt}(v)-\operatorname{wt}(w)} \otimes w \otimes v+M_{\lambda-\operatorname{wt}(v)-\operatorname{wt}(w)}[<\lambda-\operatorname{wt}(v)-\operatorname{wt}(w)] \otimes W \otimes V \\
& \quad+x_{\lambda-\operatorname{wt}(v)-\operatorname{wt}(w)} \otimes W[<\operatorname{wt}(w)] \otimes V[>\operatorname{wt}(v)] \\
& \quad+M_{\lambda-\operatorname{wt}(v)-\operatorname{wt}(w)}[<\lambda-\operatorname{wt}(v)-\operatorname{wt}(w)] \otimes W \otimes V .
\end{aligned}
$$

Hence

$$
J_{W V}(\lambda)(w \otimes v) \in w \otimes v+W[<\operatorname{wt}(w)] \otimes V[>\operatorname{wt}(v)]
$$

Proof Of [E-S], Proposition 2.3, PART 3. On the one hand we have

$$
\begin{gathered}
\left(\Phi_{\lambda-\mathrm{wt}(v)-\mathrm{wt}(w)}^{u} \otimes 1 \otimes 1\right) \circ\left(\Phi_{\lambda-\mathrm{wt}(v)}^{w} \otimes 1\right) \circ \Phi_{\lambda}^{v}\left(x_{\lambda}\right) \\
=\left(\Phi_{\lambda-\mathrm{wt}(v)-\mathrm{wt}(w)}^{u} \otimes 1 \otimes 1\right) \circ \Phi_{\lambda}^{J_{W V}(\lambda)(w \otimes v)}\left(x_{\lambda}\right) \\
=\Phi_{\lambda}^{J_{U, W \otimes V}(\lambda) \circ\left(1 \otimes J_{W V}(\lambda)\right)(u \otimes w \otimes v)}\left(x_{\lambda}\right) .
\end{gathered}
$$

On the other hand, expression (3.5) also equals

$$
\left(\Phi_{\lambda-\operatorname{wt}(v)}^{J_{U W}(\lambda-\mathrm{wt}(v))(u \otimes w)} \otimes 1\right) \circ \Phi_{\lambda}^{v}\left(x_{\lambda}\right)=\Phi_{\lambda}^{\left(J_{U \otimes W, V} \otimes 1\right)(\lambda) \circ J_{U W}(\lambda-\operatorname{wt}(v))(u \otimes w \otimes v)}\left(x_{\lambda}\right) .
$$

Hence, by equality of expressions (3.6) and (3.7), we have

$$
J_{U, W \otimes V}(\lambda) \circ\left(1 \otimes J_{W V}(\lambda)\right)(u \otimes w \otimes v)=\left(J_{U \otimes W, V} \otimes 1\right)(\lambda) \circ J_{U W}(\lambda-\operatorname{wt}(v))(u \otimes w \otimes v) .
$$


Hence we arrive at the dynamical 2-cocycle condition, which was to be proved:

$$
J_{U, W \otimes V}(\lambda) \circ\left(1 \otimes J_{W V}(\lambda)\right)=\left(J_{U \otimes W, V} \otimes 1\right)(\lambda) \circ J_{U W}\left(\lambda-h^{(3)}\right) .
$$

4. The exchange matrix. Proposition 2.4 in [E-S] states that the exchange matrix $R_{V W}(\lambda):=J_{V W}(\lambda)^{-1} J_{W V}^{21}(\lambda)$ satisfies the QDYBE

$$
R_{V W}\left(\lambda-h^{(3)}\right) R_{V U}(\lambda) R_{W U}\left(\lambda-h^{(1)}\right)=R_{W U}(\lambda) R_{V U}\left(\lambda-h^{(2)}\right) R_{V W}(\lambda)
$$

as an identity of operators on $V \otimes W \otimes U$.

In preparation of the proof recall that $\Phi_{\lambda}^{w, v}:=\left(\Phi_{\lambda-\mathrm{wt}(v)}^{w} \otimes 1\right) \circ \Phi_{\lambda}^{v}$. We have the following.

LEMMA $4.1[\mathrm{E}-\mathrm{S}] . \quad R_{V W}(\lambda)(v \otimes w)=\sum_{i} v_{i} \otimes w_{i}$, where $\Phi_{\lambda}^{w, v}=(1 \otimes P) \sum_{i} \Phi_{\lambda}^{v_{i}, w_{i}}$.

Proof. Assume $R_{V W}(\lambda)(v \otimes w)=\sum_{i} v_{i} \otimes w_{i}$. Then

$$
\begin{aligned}
\Phi_{\lambda}^{w, v} & =\Phi_{\lambda}^{J_{W V}(\lambda)(w \otimes v)}=\Phi_{\lambda}^{P J_{V W}(\lambda) R_{V W}(\lambda)(v \otimes w)} \\
& =(1 \otimes P) \Phi_{\lambda}^{J_{V W}(\lambda) R_{V W}(\lambda)(v \otimes w)}=(1 \otimes P) \sum_{i} \Phi_{\lambda}^{J_{V W}(\lambda)\left(v_{i} \otimes w_{i}\right)} \\
& =(1 \otimes P) \sum_{i} \Phi_{\lambda}^{v_{i}, w_{i}} .
\end{aligned}
$$

FIRST PROOF OF QDYBE (4.1). Put

$$
\begin{aligned}
\Phi_{\lambda}^{u, w, v} & :=\left(\Phi_{\lambda-\operatorname{wt}(v)-\operatorname{wt}(w)}^{u} \otimes 1 \otimes 1\right) \circ\left(\Phi_{\lambda-\operatorname{wt}(v)}^{w} \otimes 1\right) \circ \Phi_{\lambda}^{v} \\
& =\left(\Phi_{\lambda-\operatorname{wt}(v)}^{u, w} \otimes 1\right) \circ \Phi_{\lambda}^{v}=\left(\Phi_{\lambda-\operatorname{wt}(v)-\operatorname{wt}(w)}^{u} \otimes 1 \otimes 1\right) \circ \Phi_{\lambda}^{w, v}
\end{aligned}
$$

Now we have on the one hand

$$
\begin{aligned}
\Phi_{\lambda}^{u, w, v} & =\left(\Phi_{\lambda-\operatorname{wt}(v)-\operatorname{wt}(w)}^{u} \otimes 1 \otimes 1\right) \circ \Phi_{\lambda}^{w, v} \\
& =P^{34} \sum_{i}\left(\Phi_{\lambda-\operatorname{wt}\left(v_{i}\right)-\mathrm{wt}\left(w_{i}\right)}^{u} \otimes 1 \otimes 1\right) \circ \Phi_{\lambda}^{v_{i}, w_{i}} \\
& =P^{34} \sum_{i}\left(\Phi_{\lambda-\mathrm{wt}\left(w_{i}\right)}^{u, v_{i}} \otimes 1\right) \circ \Phi_{\lambda}^{w_{i}} \\
& =P^{34} P^{23} \sum_{i} \sum_{j}\left(\Phi_{\lambda-\mathrm{wt}\left(w_{i}\right)}^{\left(v_{i}\right)_{j}, u_{j}} \otimes 1\right) \circ \Phi_{\lambda}^{w_{i}} \\
& =P^{34} P^{23} \sum_{i} \sum_{j}\left(\Phi_{\lambda-\mathrm{wt}\left(w_{i}\right)-\mathrm{wt}\left(u_{j}\right)}^{\left(v_{i}\right)_{j}} \otimes 1 \otimes 1\right) \circ \Phi_{\lambda}^{u_{j}, w_{i}} \\
& =P^{34} P^{23} P^{34} \sum_{i} \sum_{j} \sum_{k}\left(\Phi_{\lambda-\mathrm{wt}\left(\left(w_{i}\right)_{k}\right)-\mathrm{wt}\left(\left(u_{j}\right)_{k}\right)}^{\left(v_{i}\right)_{j}} \otimes 1 \otimes 1\right) \circ \Phi_{\lambda}^{\left(w_{i}\right)_{k},\left(u_{j}\right)_{k}} \\
& =P^{34} P^{23} P^{34} \sum_{i} \sum_{j} \sum_{k} \Phi_{\lambda}^{\left(v_{i}\right)_{j},\left(w_{i}\right)_{k},\left(u_{j}\right)_{k}},
\end{aligned}
$$


and accordingly

$$
\begin{aligned}
R_{W U}(\lambda) R_{V U}\left(\lambda-h^{(2)}\right) R_{V W}(\lambda)(v \otimes w \otimes u) & =\sum_{i} R_{W U}(\lambda) R_{V U}\left(\lambda-h^{(2)}\right)\left(v_{i} \otimes w_{i} \otimes u\right) \\
& =\sum_{i} R_{W U}(\lambda) R_{V U}\left(\lambda-\operatorname{wt}\left(w_{i}\right)\right)\left(v_{i} \otimes w_{i} \otimes u\right) \\
& =\sum_{i} \sum_{j} R_{W U}(\lambda)\left(\left(v_{i}\right)_{j} \otimes w_{i} \otimes u_{j}\right) \\
& =\sum_{i} \sum_{j} \sum_{k}\left(v_{i}\right)_{j} \otimes\left(w_{i}\right)_{k} \otimes\left(u_{j}\right)_{k} .
\end{aligned}
$$

On the other hand, we have

$$
\begin{aligned}
\Phi_{\lambda}^{u, w, v} & =\left(\Phi_{\lambda-\operatorname{wt}(v)}^{u, w} \otimes 1\right) \circ \Phi_{\lambda}^{v}=P^{23} \sum_{i}\left(\Phi_{\lambda-\operatorname{wt}(v)}^{w_{i}, v_{i}} \otimes 1\right) \circ \Phi_{\lambda}^{v} \\
& =P^{23} \sum_{i}\left(\Phi_{\lambda-\operatorname{wt}(v)-\operatorname{wt}\left(u_{i}\right)}^{w_{i}} \otimes 1 \otimes 1\right) \circ \Phi_{\lambda}^{u_{i}, v} \\
& =P^{23} P^{34} \sum_{i} \sum_{j}\left(\Phi_{\lambda-\operatorname{wt}\left(v_{j}\right)-\operatorname{wt}\left(\left(u_{i}\right)_{j}\right)}^{w_{i}} \otimes 1 \otimes 1\right) \circ \Phi_{\lambda}^{v_{j},\left(u_{i}\right)_{j}} \\
& =P^{23} P^{34} \sum_{i} \sum_{j}\left(\Phi_{\lambda-\operatorname{wt}\left(\left(u_{i}\right)_{j}\right)}^{w_{i}, v_{j}} \otimes 1\right) \circ \Phi_{\lambda}^{\left(u_{i}\right)_{j}} \\
& =P^{23} P^{34} P^{23} \sum_{i} \sum_{j} \sum_{k}\left(\Phi_{\lambda-\operatorname{wt}\left(\left(u_{i}\right)_{j}\right)}^{\left(v_{j}\right)_{k},\left(w_{i}\right)_{k}} \otimes 1\right) \circ \Phi_{\lambda}^{\left(u_{i}\right)_{j}} \\
& =P^{23} P^{34} P^{23} \sum_{i} \sum_{j} \sum_{k} \Phi_{\lambda}^{\left(v_{j}\right)_{k},\left(w_{i}\right)_{k},\left(u_{i}\right)_{j}},
\end{aligned}
$$

and accordingly

$$
\begin{aligned}
R_{V W}(\lambda & \left.-h^{(3)}\right) R_{V U}(\lambda) R_{W U}\left(\lambda-h^{(1)}\right)(v \otimes w \otimes u) \\
& =R_{V W}\left(\lambda-h^{(3)}\right) R_{V U}(\lambda) R_{W U}(\lambda-\mathrm{wt}(v))(v \otimes w \otimes u) \\
& =\sum_{i} R_{V W}\left(\lambda-h^{(3)}\right) R_{V U}(\lambda)\left(v \otimes w_{i} \otimes u_{i}\right) \\
& =\sum_{i} \sum_{j} R_{V W}\left(\lambda-h^{(3)}\right)\left(v_{j} \otimes w_{i} \otimes\left(u_{i}\right)_{j}\right) \\
& =\sum_{i} \sum_{j} R_{V W}\left(\lambda-\operatorname{wt}\left(\left(u_{i}\right)_{j}\right)\right)\left(v_{j} \otimes w_{i} \otimes\left(u_{i}\right)_{j}\right) \\
& =\sum_{i} \sum_{j} \sum_{k}\left(v_{j}\right)_{k} \otimes\left(w_{i}\right)_{k} \otimes\left(u_{i}\right)_{j}
\end{aligned}
$$

It follows from (4.4) and (4.6) that

$$
\sum_{i} \sum_{j} \sum_{k} \Phi_{\lambda}^{\left(v_{i}\right)_{j},\left(w_{i}\right)_{k},\left(u_{j}\right)_{k}}=\sum_{i} \sum_{j} \sum_{k} \Phi_{\lambda}^{\left(v_{j}\right)_{k},\left(w_{i}\right)_{k},\left(u_{i}\right)_{j}} .
$$

Hence the right-hand sides of (4.5) and (4.7) are equal. Thus the left-hand sides of (4.5) and (4.7) are also equal. 
As pointed out in [E-S], Section 2.2 the construction of intertwining operators, fusion and exchange matrices admit natural quantum analogues. Most definitions, results and proofs go on essentially unchanged compared to the $q=1$ case. However, in the definition of the exchange matrix the $R$-matrix $\mathscr{R}_{V W}$ associated to $U_{q}(\mathfrak{g})$-modules $V$ and $W$, and induced by the universal $R$-matrix $\mathscr{R}$, is also needed. I will use the notation

$$
\mathscr{R}_{W V}^{21}:=\left(\mathscr{R}_{W V}\right)^{21}=P_{W V} \mathscr{R}_{W V} P_{V W} .
$$

This is different from the notation $\mathscr{R}_{V W}^{21}:=\left(\mathscr{R}^{21}\right)_{V W}$ in [E-S], Section 2.2. The exchange matrix in the quantum case is now defined by

$$
R_{V W}(\lambda):=J_{V W}(\lambda)^{-1} \mathscr{R}_{W V}^{21} J_{W V}^{21}(\lambda) .
$$

The dynamical two-cocycle condition (3.9) will remain valid in the quantum case. I will now discuss a second proof of the QDYBE (4.1), which is briefly sketched in the remark in [E-S] after Proposition 2.4, and which also holds in the quantum case. In the following, when being in the $q=1$ case, just put $\mathscr{R}_{V W}$ equal to 1 (for any $V, W$ ).

I derive first the following two important formulas (not given in [E-S]) for the exchange matrix:

$$
\begin{aligned}
& J_{V W}(\lambda)^{-1} R_{U, V \otimes W}(\lambda) J_{V W}\left(\lambda-h^{(U)}\right)=R_{U V}\left(\lambda-h^{(W)}\right) R_{U W}(\lambda), \\
& J_{U V}\left(\lambda-h^{(W)}\right)^{-1} R_{U \otimes V, W}(\lambda) J_{U V}(\lambda)=R_{V W}(\lambda) R_{U W}\left(\lambda-h^{(V)}\right),
\end{aligned}
$$

where both sides in (4.11) and (4.12) are acting on $U \otimes V \otimes W$. Here we have adapted the notation introduced in [E-S] just before Proposition 2.3 as follows. If $U=A_{i}$ then $F\left(\lambda-h^{(U)}\right)$ will mean $F\left(\lambda-h^{(i)}\right)$.

One of the formulas (4.11) and (4.12) can be obtained by specialization of formula (2.42) in [E-V]. Note that (4.11) and (4.12) are also dynamical analogues of the formulas

$$
\mathscr{R}_{U \otimes V, W}=\mathscr{R}_{U W} \mathscr{R}_{V W}, \quad \mathscr{R}_{U, V \otimes W}=\mathscr{R}_{U W} \mathscr{R}_{U V},
$$

obtained from the following formulas for the universal $R$-matrix:

$$
(\Delta \otimes \mathrm{id})(\mathscr{R})=\mathscr{R}_{13} \mathscr{R}_{23}, \quad(\mathrm{id} \otimes \Delta)(\mathscr{R})=\mathscr{R}_{13} \mathscr{R}_{12},
$$

which belong to the defining properties of a quasitriangular Hopf algebra. Another defining property of a quasitriangular Hopf algebra is that

$$
P(\Delta(u))=\mathscr{R} \Delta(u) \mathscr{R}^{-1},
$$

which implies for the universal fusion matrix $J(\lambda)$ (see [E-S], Section 8) that

$$
\begin{gathered}
P_{12}(\Delta \otimes 1)(J(\lambda))=\mathscr{R}_{12}(\Delta \otimes 1)(J(\lambda)) \mathscr{R}_{12}^{-1}, \\
P_{23}(1 \otimes \Delta)(J(\lambda))=\mathscr{R}_{23}(1 \otimes \Delta)(J(\lambda)) \mathscr{R}_{23}^{-1},
\end{gathered}
$$

and hence

$$
\begin{aligned}
& P_{W V} J_{W \otimes V, U}(\lambda) P_{V W}=\mathscr{R}_{V W} J_{V \otimes W, U}(\lambda) \mathscr{R}_{V W}^{-1}, \\
& P_{U W} J_{V, U \otimes W}(\lambda) P_{W U}=\mathscr{R}_{W U} J_{V, W \otimes U}(\lambda) \mathscr{R}_{W U}^{-1} .
\end{aligned}
$$

In the proof of (4.11) and (4.12) I need (4.13) and (4.17). 


\section{PROOF OF (4.11).}

$$
\begin{aligned}
& J_{V W}(\lambda)^{-1} R_{U, V \otimes W}(\lambda) J_{V W}\left(\lambda-h^{(U)}\right) \\
& \quad=J_{V W}(\lambda)^{-1} J_{U, V \otimes W}(\lambda)^{-1} J_{V \otimes W, U}^{21}(\lambda) \mathscr{R}_{V \otimes W, U}^{21} J_{V W}\left(\lambda-h^{(U)}\right) \\
& =J_{U V}\left(\lambda-h^{(W)}\right)^{-1} J_{U \otimes V, W}(\lambda)^{-1} P_{V U} P_{W U} \mathscr{R}_{V \otimes W, U} J_{V \otimes W, U}(\lambda) P_{U W} P_{U V} J_{V W}\left(\lambda-h^{(U)}\right) \\
& =J_{U V}\left(\lambda-h^{(W)}\right)^{-1} J_{U \otimes V, W}(\lambda)^{-1} P_{V U} P_{W U} \mathscr{R}_{V U} \Re_{W U} J_{V \otimes W, U}(\lambda) J_{V W}\left(\lambda-h^{(U)}\right) P_{U W} P_{U V} \\
& =J_{U V}\left(\lambda-h^{(W)}\right)^{-1} J_{U \otimes V, W}(\lambda)^{-1} P_{V U} \mathscr{R}_{V U} P_{W U} \mathscr{R}_{W U} J_{V, W \otimes U}(\lambda) J_{W U}(\lambda) P_{U W} P_{U V} \\
& =J_{U V}\left(\lambda-h^{(W)}\right)^{-1} P_{V U} \mathscr{R}_{V U} J_{V \otimes U, W}(\lambda)^{-1} J_{V, U \otimes W}(\lambda) P_{W U} \mathscr{R}_{W U} J_{W U}(\lambda) P_{U W} P_{U V} \\
& =J_{U V}\left(\lambda-h^{(W)}\right)^{-1} P_{V U} \mathscr{R}_{V U} J_{V U}\left(\lambda-h^{(W)}\right) J_{U W}(\lambda)^{-1} P_{W U} \mathscr{R}_{W U} J_{W U}(\lambda) P_{U W} P_{U V} \\
& =J_{U V}\left(\lambda-h^{(W)}\right)^{-1} \mathscr{R}_{V U}^{21} J_{V U}^{21}\left(\lambda-h^{(W)}\right) P_{V U} J_{U W}(\lambda)^{-1} \mathscr{R}_{W U}^{21} J_{W U}^{21}(\lambda) P_{U V} \\
& =R_{U V}\left(\lambda-h^{(W)}\right) R_{U W}(\lambda) .
\end{aligned}
$$

\section{PROOF OF (4.12).}

$$
\begin{aligned}
J_{U V} & \left(\lambda-h^{(W)}\right)^{-1} R_{U \otimes V, W}(\lambda) J_{U V}(\lambda) \\
& =J_{U V}\left(\lambda-h^{(W)}\right)^{-1} J_{U \otimes V, W}(\lambda)^{-1} \mathscr{R}_{W, U \otimes V}^{21} J_{W, U \otimes V}^{21}(\lambda) J_{U V}(\lambda) \\
& =J_{V W}(\lambda)^{-1} J_{U, V \otimes W}(\lambda)^{-1} P_{W V} P_{W U} \Re_{W, U \otimes V} J_{W, U \otimes V}(\lambda) P_{U W} P_{V W} J_{U V}(\lambda) \\
& =J_{V W}(\lambda)^{-1} J_{U, V \otimes W}(\lambda)^{-1} P_{W V} P_{W U} \Re_{W V} \mathscr{R}_{W U} J_{W, U \otimes V}(\lambda) J_{U V}(\lambda) P_{U W} P_{V W} \\
& =J_{V W}(\lambda)^{-1} J_{U, V \otimes W}(\lambda)^{-1} P_{W V} \mathscr{R}_{W V} P_{W U} \mathscr{R}_{W U} J_{W \otimes U, V}(\lambda) J_{W U}\left(\lambda-h^{(V)}\right) P_{W U} P_{W V} \\
& =J_{V W}(\lambda)^{-1} P_{W V} \Re_{W V} J_{U, W \otimes V}(\lambda)^{-1} J_{U \otimes W, V}(\lambda) P_{W U} \Re_{W U} J_{W U}\left(\lambda-h^{(V)}\right) P_{U W} P_{V W} \\
& =J_{V W}(\lambda)^{-1} P_{W V} \mathscr{R}_{W V} J_{W V}(\lambda) J_{U W}\left(\lambda-h^{(V)}\right)^{-1} P_{W U} \mathscr{R}_{W U} J_{W U}\left(\lambda-h^{(V)}\right) P_{U W} P_{V W} \\
& =J_{V W}(\lambda)^{-1} \Re_{W V}^{21} J_{W V}^{21}(\lambda) P_{W V} J_{U W}\left(\lambda-h^{(V)}\right)^{-1} \Re_{W U}^{21} J_{W U}^{21}\left(\lambda-h^{(V)}\right) P_{V W} \\
& =R_{V W}(\lambda) R_{U W}\left(\lambda-h^{(V)}\right) .
\end{aligned}
$$

In both proofs we have used the 2-cocycle condition (3.9) for the fusion matrix three times.

SECOND PROOF OF QDYBE (4.1) (USING (4.11) AND (4.12); ACTING ON $V \otimes W \otimes U$ ).

$$
\begin{aligned}
& R_{V W}\left(\lambda-h^{(U)}\right) R_{V U}(\lambda) R_{W U}\left(\lambda-h^{(V)}\right) \\
& \quad=J_{W U}(\lambda)^{-1} R_{V, W \otimes U}(\lambda) J_{W U}\left(\lambda-h^{(V)}\right) J_{W U}\left(\lambda-h^{(V)}\right)^{-1} \mathscr{R}_{U W}^{21} J_{U W}^{21}\left(\lambda-h^{(V)}\right) \\
& \quad=J_{W U}(\lambda)^{-1} R_{V, W \otimes U}(\lambda) P_{U W} \mathscr{R}_{U W} J_{U W}\left(\lambda-h^{(V)}\right) P_{W U} \\
& \quad=J_{W U}(\lambda)^{-1} P_{U W} \mathscr{R}_{U W} R_{V, U \otimes W}(\lambda) J_{U W}\left(\lambda-h^{(V)}\right) P_{W U} \\
& =J_{W U}(\lambda)^{-1} P_{U W} \mathscr{R}_{U W} J_{U W}(\lambda) R_{V U}\left(\lambda-h^{(W)}\right) R_{V W}(\lambda) P_{W U} \\
& =R_{W U}(\lambda) P_{U W} R_{V U}\left(\lambda-h^{(W)}\right) R_{V W}(\lambda) P_{W U} \\
& =R_{W U}(\lambda) R_{V U}\left(\lambda-h^{(W)}\right) R_{V W}(\lambda) .
\end{aligned}
$$


5. Difference operators. Next I give a proof for the $q=1$ case of the formula

$$
\mathscr{D}_{V \otimes W}^{U}=\mathscr{D}_{V}^{U} \mathscr{D}_{W}^{U}=\mathscr{D}_{W}^{U} \mathscr{D}_{V}^{U} \text {, }
$$

stated at the end of Section 9.1 in [E-S] for the quantum case. Let $\mathfrak{g}$ be a simple Lie algebra. For any two finite-dimensional $\mathfrak{g}$-modules $U$ and $V$ let $R_{V U}(\lambda)$ be the exchange matrix. Let $\mathbb{R}_{V U}(\lambda):=R_{V U}(-\lambda-\rho)$ denote the shifted exchange matrix. Let $\mathscr{F}_{U}$ be the space of $U[0]$-valued meromorphic functions on $\mathfrak{h}^{*}$. For $v \in \mathfrak{h}^{*}$ let $T_{v} \in \operatorname{End}\left(\mathscr{F}_{U}\right)$ be the shift operator $\left(T_{v} f\right)(\lambda):=f(\lambda+\nu)$. Define the difference operator $\mathscr{D}_{V}^{\lambda, U}$ acting on $\mathscr{F}_{U}$ by

$$
\mathscr{D}_{V}^{\lambda, U}:=\left.\sum_{v \in \mathfrak{h}^{*}} \operatorname{Tr}\right|_{V[v]}\left(\mathbb{R}_{V U}(\lambda)\right) T_{v}=\left.\sum_{v \in \mathfrak{h}^{*}} \operatorname{Tr}\right|_{V[v]}\left(\mathbb{R}_{V[v], U[0] ; V[v], U[0]}(\lambda)\right) T_{v},
$$

where $\mathbb{R}_{V[\lambda], U[\mu] ; V[v], U[\sigma]}$ denotes the block of the matrix $\mathbb{R}_{V U}$ corresponding to the weight spaces $V[\lambda], U[\mu] ; V[v], U[\sigma]$ (which block will be zero unless $\lambda+\mu=\nu+\sigma$ ).

Proof OF (5.1). We can rewrite (4.12) as

$$
\mathbb{R}_{W \otimes V, U}(\lambda)=\rrbracket_{W V}\left(\lambda+h^{(U)}\right) \mathbb{R}_{V U}(\lambda) \mathbb{R}_{W U}\left(\lambda+h^{(V)}\right) \rrbracket_{W V}(\lambda)^{-1},
$$

where $\rrbracket_{W V}(\lambda):=J(-\lambda-\rho)$ denotes the shifted fusion matrix. Hence

$$
\begin{aligned}
\mathbb{R}_{W[v] \otimes V[\mu], U[0] ; W[v] \otimes V[\mu], U[0]}(\lambda)= & \sum_{\mu^{\prime}, v^{\prime}, \mu^{\prime \prime}, v^{\prime \prime}, \sigma} \mathbb{I}_{W[v], V[\mu] ; W\left[\nu^{\prime}\right], V\left[\mu^{\prime}\right]}(\lambda) \\
& \circ \mathbb{R}_{V\left[\mu^{\prime}\right], U[0] ; V\left[\mu^{\prime \prime}\right], U[\sigma]}(\lambda) \mathbb{R}_{W\left[\nu^{\prime}\right], U[\sigma] ; W\left[v^{\prime \prime}\right], U[0]}\left(\lambda+\mu^{\prime \prime}\right) \\
& \times \rrbracket_{W[\nu], V[\mu] ; W\left[\nu^{\prime \prime}\right], V\left[\mu^{\prime \prime}\right]}(\lambda)^{-1} .
\end{aligned}
$$

Hence

$$
\begin{aligned}
& \left.\operatorname{Tr}\right|_{W[v] \otimes V[\mu]}\left(\mathbb{R}_{W[v] \otimes V[\mu], U[0] ; W[v] \otimes V[\mu], U[0]}(\lambda)\right) \\
& \quad=\left.\sum_{\sigma} \operatorname{Tr}\right|_{W[v] \otimes V[\mu]}\left(\mathbb{R}_{V[\mu], U[0] ; V[\mu], U[\sigma]}(\lambda) \mathbb{R}_{W[v], U[\sigma] ; W[v], U[0]}(\lambda+\mu)\right) \\
& \quad=\left.\operatorname{Tr}\right|_{W[v] \otimes V[\mu]}\left(\mathbb{R}_{V[\mu], U[0] ; V[\mu], U[0]}(\lambda) \mathbb{R}_{W[v], U[0] ; W[v], U[0]}(\lambda+\mu)\right) .
\end{aligned}
$$

Then

$$
\begin{aligned}
\mathscr{D}_{V}^{\lambda, U} \mathscr{D}_{W}^{\lambda, U} & =\left.\left.\sum_{\mu} \operatorname{Tr}\right|_{V[\mu]}\left(\mathbb{R}_{V[\mu], U[0] ; V[\mu], U[0]}(\lambda)\right) T_{\mu} \sum_{v} \operatorname{Tr}\right|_{W[v]}\left(\mathbb{R}_{W[v], U[0] ; W[v], U[0]}(\lambda)\right) T_{v} \\
& =\left.\left.\sum_{\mu, v} \operatorname{Tr}\right|_{V[\mu]}\left(\mathbb{R}_{V[\mu], U[0] ; V[\mu], U[0]}(\lambda)\right) \operatorname{Tr}\right|_{W[v]}\left(\mathbb{R}_{W[v], U[0] ; W[v], U[0]}(\lambda+\mu)\right) T_{\mu+v} \\
& =\left.\sum_{\mu, v} \operatorname{Tr}\right|_{W[v] \otimes V[\mu]}\left(\mathbb{R}_{V[\mu], U[0] ; V[\mu], U[0]}(\lambda) \mathbb{R}_{W[v], U[0] ; W[v], U[0]}(\lambda+\mu)\right) T_{\mu+v} \\
& =\left.\sum_{\mu, v} \operatorname{Tr}\right|_{W[v] \otimes V[\mu]}\left(\mathbb{R}_{W[v] \otimes V[\mu], U[0] ; W[v] \otimes V[\mu], U[0]}(\lambda)\right) T_{\mu+v} \\
& =\left.\sum_{\sigma} \operatorname{Tr}\right|_{(W \otimes V)[\sigma]}\left(\mathbb{R}_{(W \otimes V)[\sigma], U[0] ;(W \otimes V)[\sigma], U[0]}(\lambda)\right) T_{\sigma}=\mathscr{D}_{W \otimes V}^{\lambda, U} .
\end{aligned}
$$


But also

$$
\begin{aligned}
\mathscr{D}_{W \otimes V}^{\lambda, U}= & \left.\sum_{\mu, v} \operatorname{Tr}\right|_{W[v] \otimes V[\mu]}\left(\mathbb{R}_{W[v] \otimes V[\mu], U[0] ; W[v] \otimes V[\mu], U[0]}(\lambda)\right) T_{\mu+v} \\
=\left.\sum_{\mu, v} \operatorname{Tr}\right|_{W[v] \otimes V[\mu]}( & P_{V[\mu], W[v] ; V[\mu], W[v]} \mathbb{R}_{V[\mu] \otimes W[v], U[0] ; V[\mu] \otimes W[v], U[0]}(\lambda) \\
& \left.\times P_{W[v], V[\mu] ; W[v], V[\mu]}\right) \circ T_{\mu+v} \\
= & \left.\sum_{\mu, v} \operatorname{Tr}\right|_{V[\mu] \otimes W[v]}\left(\mathbb{R}_{V[\mu] \otimes W[v], U[0] ; V[\mu] \otimes W[v], U[0]}(\lambda)\right) T_{\mu+v}=\mathscr{D}_{V \otimes W}^{\lambda, U} .
\end{aligned}
$$

Hence

$$
\mathscr{D}_{V}^{\lambda, U} \mathscr{D}_{W}^{\lambda, U}=\mathscr{D}_{W \otimes V}^{\lambda, U}=\mathscr{D}_{V \otimes W}^{\lambda, U}=\mathscr{D}_{W}^{\lambda, U} \mathscr{D}_{V}^{\lambda, U} .
$$

Note that it was possible to apply (4.12) in the above proof because we had assumed that $\mathscr{D}_{V}^{\lambda, U}$ acts on $U[0]$-valued functions, and because the definition of $\mathscr{D}_{V}^{\lambda, U}$ involved shift operators $T_{v}$.

6. Weighted trace functions. In Section 9.2 of [E-S] weighted-trace functions are introduced and difference equations are given for them. [E-S] refers for the proofs to [E-V]. Theorem 9.2 of [E-S] survives for $q=1$, see [E-V], Theorem 10.4. I will give a proof of that theorem parallel to the proof of the $q$-case, see Theorem 1.2 and Section 3 in [E-V].

First consider the proof of Lemma 2.14 in [E-V]. Let $W$ be a finite-dimensional $\mathfrak{g}$ module. By the properties of the intertwining operator we can uniquely define a bilinear form $B_{\lambda, W}: W \times W^{*} \rightarrow \mathbb{C}$ by the formula

$$
(1 \otimes\langle,\rangle) \circ\left(\Phi_{\lambda-\operatorname{wt}\left(w^{*}\right)}^{w} \otimes 1\right) \circ \Phi_{\lambda}^{w^{*}}=B_{\lambda, W}\left(w, w^{*}\right) \operatorname{id}_{M_{\lambda}} .
$$

Note that $B_{\lambda, W}\left(w, w^{*}\right)=0$ if $\operatorname{wt}(w)+\operatorname{wt}\left(w^{*}\right) \neq 0$. Since

$$
\Phi_{\lambda}^{J_{W W^{*}(\lambda)\left(w \otimes w^{*}\right)}}=\left(\Phi_{\lambda-\mathrm{wt}\left(w^{*}\right)}^{w} \otimes 1\right) \circ \Phi_{\lambda}^{w^{*}},
$$

we have

$$
B_{\lambda, W}\left(w, w^{*}\right)=\langle,\rangle\left(J_{W W^{*}}(\lambda)\left(w \otimes w^{*}\right)\right) .
$$

Define a generalized element $Q(\lambda)$ in $U(\mathfrak{g})$ in terms of the universal fusion matrix by

$$
Q(\lambda):=\left(m \circ P \circ\left(1 \otimes S^{-1}\right)\right) J(\lambda) .
$$

This induces an endomorphism $Q_{W}(\lambda)$ of $W$ given by

$$
Q_{W}(\lambda)=C_{W}\left(\left(J_{W W^{*}}(\lambda)^{t_{2}}\right)^{21}\right),
$$

where $C_{W}$ denotes contraction of an endomorphism of $W \otimes W$ to an endomorphism of $W$. Now we have

$$
B_{\lambda, W}\left(w, w^{*}\right)=\left\langle Q_{W}(\lambda) w, w^{*}\right\rangle .
$$


Indeed, if $T \in \operatorname{End}(W \otimes W)$ then $\left\langle C(T) w, w^{*}\right\rangle=\langle\rangle,\left(\left(T^{21}\right)^{t_{2}}\left(w \otimes w^{*}\right)\right)$. Hence

$$
\left\langle Q_{W}(\lambda) w, w^{*}\right\rangle=\langle,\rangle\left(J_{W W^{*}}(\lambda)\left(w \otimes w^{*}\right)\right)=B_{\lambda, W}\left(w, w^{*}\right) .
$$

It follows from (6.6) that $Q_{W}(\lambda)$ is a weight preserving endomorphism of $W$.

Next we have

$$
B_{\lambda, U \otimes W} \circ\left(J_{U W}\left(\lambda-h^{\left(U^{*}\right)}-h^{\left(W^{*}\right)}\right) \otimes J_{U^{*} W^{*}}(\lambda)\right)=B_{\lambda, U} \circ B_{\lambda-h^{\left(U^{*}\right)}, W} .
$$

Indeed,

$$
\begin{aligned}
& B_{\lambda, U}\left(u, u^{*}\right) B_{\lambda-w t}\left(u^{*}\right), W \operatorname{id}_{M_{\lambda}} \\
& =(\langle,\rangle \otimes\langle,\rangle) \circ \Phi_{\lambda-\mathrm{wt}\left(u^{*}\right)-\mathrm{wt}\left(w^{*}\right)-\mathrm{wt}(w)}^{u} \circ \Phi_{\lambda-\mathrm{wt}\left(u^{*}\right)-\mathrm{wt}\left(w^{*}\right)}^{w} \circ \Phi_{\lambda-\mathrm{wt}\left(u^{*}\right)}^{w^{*}} \circ \Phi_{\lambda}^{u^{*}} \\
& =(\langle,\rangle \otimes\langle,\rangle) \circ \Phi_{\lambda-\operatorname{wt}\left(u^{*}\right)-\operatorname{wt}\left(w^{*}\right)}^{J_{U W}\left(\left(w^{*}\right)\right)(u \otimes w)} \circ \Phi_{\lambda}^{J_{W^{*}} U^{*}(\lambda)\left(w^{*} \otimes u^{*}\right)} \\
& =B_{\lambda, U \otimes W}\left(J_{U W}\left(\lambda-\operatorname{wt}\left(u^{*}\right)-\text { wt }\left(w^{*}\right)\right)(u \otimes w), J_{W^{*} U^{*}}^{21}(\lambda)\left(u^{*} \otimes w^{*}\right)\right) \operatorname{id}_{M_{\lambda}} .
\end{aligned}
$$

Combination of (6.6) with (6.8) yields that

$$
Q_{U \otimes W}(\lambda)=\left(J_{W^{*} U^{*}}^{t_{1} t_{2}, 21}(\lambda)\right)^{-1}\left(Q_{U}(\lambda) \otimes Q_{W}\left(\lambda+h^{(U)}\right)\right) J_{U W}\left(\lambda+h^{(U)}+h^{(W)}\right)^{-1} .
$$

It follows from (6.1) and (6.6) that $Q_{U \otimes W}(\lambda)=Q_{W \otimes U}^{21}(\lambda)$. Hence we can rewrite (6.10) as

$$
Q_{U \otimes W}(\lambda)=\left(J_{U^{*} W^{*}}^{t_{1} t_{2}}(\lambda)\right)^{-1}\left(Q_{U}\left(\lambda+h^{(W)}\right) \otimes Q_{W}(\lambda)\right) J_{W U}^{21}\left(\lambda+h^{(U)}+h^{(W)}\right)^{-1} .
$$

Now eliminate $Q_{U \otimes W}(\lambda)$ from these two formulas and substitute

$$
R_{U W}(\lambda)=J_{U W}(\lambda)^{-1} J_{W U}^{21}(\lambda)
$$

(the defining formula for the exchange matrix in Section 2.1 of [E-S]). Then we obtain $R_{U^{*} W^{*}}^{t_{1} t_{2}}(\lambda)=\left(Q_{U}(\lambda) \otimes Q_{W}\left(\lambda+h^{(U)}\right)\right) \circ R_{U W}\left(\lambda+h^{(U)}+h^{(W)}\right)\left(Q_{U}\left(\lambda+h^{(W)}\right) \otimes Q_{W}(\lambda)\right)^{-1}$.

This is essentially the formula at the end of Section 3.3 in [E-V].

Next I discuss Proposition 3.1 in [E-V]. Fix finite-dimensional $\mathfrak{g}$-modules $V$ and $W$. Let $B$ be a basis of $V$ consisting of weight vectors. For $v \in B$ let $V^{*}$ be the corresponding dual basis vector of $V^{*}$. Define the operator

$$
\Phi_{\mu}^{V}: y \longmapsto \sum_{v \in B} \Phi_{\mu}^{v}(y) \otimes v^{*}: M_{\mu} \longrightarrow \bigoplus_{\lambda}\left(M_{\mu-\lambda} \otimes V \otimes V^{*}[-\lambda]\right),
$$

which is clearly independent of the choice of $B$. Define the isomorphism

$$
\eta_{W}(\mu): \bigoplus_{v}\left(W[v] \otimes M_{\mu+v}\right) \longrightarrow M_{\mu} \otimes W
$$

where

$$
\eta_{W}(\mu)(w \otimes z):=\Phi_{\mu+v}^{w}(z) \quad \text { if } w \in W[v], z \in M_{\mu+v} .
$$

Proposition 3.1 together with formula (3.2) in [E-V] can now be formulated as follows:

$$
\begin{aligned}
& \left.P_{V \otimes V^{*}, W} \circ\left(\Phi_{\mu}^{V} \otimes \operatorname{id}_{W}\right) \circ \eta_{W}(\mu)\right|_{W[v] \otimes M_{\mu+v}} \\
& \quad=\left.\left(\eta_{W}(\mu) \otimes \operatorname{id}_{V} \otimes \operatorname{id}_{V^{*}}\right) \circ R_{W V}^{t_{2}}(\mu+\nu) \circ\left(\operatorname{id}_{W} \otimes \Phi_{\mu+v}^{V}\right)\right|_{W[v] \otimes M_{\mu+v}} .
\end{aligned}
$$


Proof OF (6.17). Write $R_{W V}(\mu+\nu)=\sum_{i} p_{i} \otimes q_{i}^{t}$. Let $y \in M_{\mu+v}$ and $w \in W[v]$. Then

$$
\begin{aligned}
\left(P_{V \otimes V^{*}, W} \circ\right. & \left.\left(\Phi_{\mu}^{V} \otimes \mathrm{id}_{W}\right) \circ \eta_{W}(\mu)\right)(w \otimes y) \\
& =\left(P_{V \otimes V^{*}, W} \circ\left(\Phi_{\mu}^{V} \otimes \mathrm{id}_{W}\right) \circ \Phi_{\mu+v}^{w}\right)(y) \\
& =P_{V W} \sum_{v \in B}\left(\Phi_{\mu}^{v} \otimes \mathrm{id}\right)\left(\Phi_{\mu+v}^{w}(y)\right) \otimes v^{*} \\
& =P_{V W} \sum_{v \in B} \Phi_{\mu+v}^{J_{V W}(\mu+v)(v \otimes w)}(y) \otimes v^{*} \\
& =\sum_{v \in B} \Phi_{\mu+v}^{J_{V W}^{21}(\mu+v)(w \otimes v)}(y) \otimes v^{*} \\
& =\sum_{v \in B} \Phi_{\mu+v}^{J_{W V}(\mu+v)\left(R_{W V}(\mu+v)(w \otimes v)\right)}(y) \otimes v^{*} \\
& =\sum_{v \in B} \sum_{i}\left(\Phi_{\mu+v-w \mathrm{t}(v)}^{p_{i} w} \otimes \operatorname{id}_{V}\right)\left(\Phi_{\mu+v}^{q_{i}^{t} v}(y)\right) \otimes v^{*} \\
& =\sum_{v \in B} \sum_{i}\left(\Phi_{\mu+v-w t(v)}^{p_{i} w} \otimes \operatorname{id}_{V}\right)\left(\Phi_{\mu+v}^{v}(y)\right) \otimes q_{i} v^{*} \\
& =\sum_{v \in B} \sum_{i}\left(\eta_{W}(\mu) \otimes \operatorname{id}_{V} \otimes \operatorname{id}_{V^{*}}\right)\left(p_{i} w \otimes \Phi_{\mu+v}^{v}(y) \otimes q_{i} v^{*}\right) \\
& =\left(\eta_{W}(\mu) \otimes \operatorname{id}_{V} \otimes \operatorname{id}_{V^{*}}\right) \circ R_{W V}^{t_{2}}(\mu+v) \circ\left(w \otimes \Phi_{\mu+v}^{V}(y)\right) .
\end{aligned}
$$

For $\lambda \in \mathfrak{h}^{*}$ and $U$ a $\mathfrak{g}$-module let $e^{\lambda}: u \mapsto e^{\langle\lambda, \mathrm{wt}(u)\rangle} u: U \rightarrow U$. Let $V$ be a finite dimensional $\mathfrak{g}$-module and let $v \in V$ [0]. Let $\left\{y_{i}\right\}$ be a basis of $M_{\mu}$ consisting of weight vectors. Since $\Phi_{\mu}^{v}: M_{\mu} \rightarrow M_{\mu} \otimes V$ is weight preserving, we have $\Phi_{\mu}^{v}\left(y_{i}\right) \in y_{i} \otimes V[0]+$ $\sum_{j \neq i} y_{j} \otimes V$. Hence, if $B[0]$ is a basis of $V[0]$ and if $v^{*} \in V^{*}[0]$ is the dual basis vector corresponding to $v \in B[0]$, we have

$$
\Phi_{\mu}^{v}\left(e^{\lambda} y_{i}\right) \otimes v^{*} \in y_{i} \otimes V[0] \otimes V^{*}[0]+\sum_{j \neq i} y_{j} \otimes V \otimes V^{*}[0] .
$$

Let

$$
\Phi_{\mu}^{V[0]}:=\sum_{v \in B[0]} \Phi_{\mu}^{v} \otimes v^{*}
$$

Then

$$
\Psi_{V}(\lambda, \mu):=\left.\operatorname{Tr}\right|_{M_{\mu}}\left(\Phi_{\mu}^{V[0]} \circ e^{\lambda}\right) \in V[0] \otimes V^{*}[0] .
$$

For $W$ a finite dimensional $\mathfrak{g}$-module let

$$
\chi_{W}\left(e^{\lambda}\right):=\left.\operatorname{Tr}\right|_{W} e^{\lambda}=\sum_{v} \operatorname{dim}(W[v]) e^{\langle\lambda, v\rangle} .
$$

A difference equation for $\Psi_{V}(\lambda, \mu)$ in the variable $\mu$ can be derived from (6.17). First multiply both sides of (6.17) with $e^{\langle\lambda, \mu+v\rangle}$, observe that $\eta_{W}(\mu) \circ\left(\operatorname{id}_{W} \otimes e^{\lambda}\right)=\left(e^{\lambda} \otimes e^{\lambda}\right) \circ$ $\eta_{W}(\mu)$, sum both sides of (6.17) with respect to $v$, and next multiply both sides of 
(6.17) on the left with $\left(\eta_{W}(\mu) \otimes \mathrm{id}_{V} \otimes \mathrm{id}_{V^{*}}\right)^{-1}$. Then we obtain the following identity of linear endomorphisms $\oplus_{\nu}\left(W[v] \otimes M_{\mu+v}\right) \rightarrow \oplus_{v}\left(W[v] \otimes M_{\mu+v} \otimes V \otimes V^{*}\right)$.

$$
\begin{aligned}
\left(\eta_{W}(\mu) \otimes \operatorname{id}_{V} \otimes \operatorname{id}_{V^{*}}\right)^{-1} \circ P_{V \otimes V^{*}, W} \circ & \left.\left(\left(\Phi_{\mu}^{V} \circ e^{\lambda}\right) \otimes\left(e^{\lambda} \circ \operatorname{id}_{W}\right)\right) \circ \eta_{W}(\mu)\right|_{\oplus v}\left(W[v] \otimes M_{\mu+v}\right) \\
& =\left.\sum_{v} R_{W V}^{t_{2}}(\mu+\nu) \circ\left(\operatorname{id}_{W} \otimes\left(\Phi_{\mu+v}^{V} \circ e^{\lambda}\right)\right)\right|_{W[v] \otimes M_{\mu+\nu}} .
\end{aligned}
$$

Now take the trace with respect to $\oplus_{\nu}\left(W[\nu] \otimes M_{\mu+v}\right)$ on both sides of (6.23) and use (6.20). Then

$$
\left(\left.\operatorname{Tr}\right|_{W} e^{\lambda}\right)\left(\left.\operatorname{Tr}\right|_{M_{\mu}} \Phi_{\mu}^{V[0]} \circ e^{\lambda}\right)=\left.\sum_{\nu} \operatorname{Tr}\right|_{W[v]} R_{W V}^{t_{2}}(\mu+v) \circ\left(\left.\operatorname{id}{ }_{W} \otimes \operatorname{Tr}\right|_{M_{\mu+\nu}} \Phi_{\mu+v}^{V[0]} \circ e^{\lambda}\right) .
$$

Now substitute (6.21) and (6.22), and take, inside the sum on the right-hand side, the transpose with respect to $W$. Then

$$
\chi_{W}\left(e^{\lambda}\right) \Psi_{V}(\lambda, \mu)=\left.\sum_{v} \operatorname{Tr}\right|_{W *[-v]} R_{W V}^{t_{1} t_{2}}(\mu+v) \circ\left(\operatorname{id}_{W *} \otimes \Psi_{V}(\lambda, \mu+v)\right) .
$$

On the right-hand side of formula (6.25) substitute (6.13). Next also substitute $\mathbb{R}_{V U}(\lambda)$ $:=R_{V U}(-\lambda-\rho)$ and $\mathbb{Q}_{V}(\lambda):=Q_{V}(-\lambda-\rho)$. Then

$$
\begin{aligned}
\chi_{W} & \left(e^{\lambda}\right) \Psi_{V}(\lambda, \mu) \\
= & \left.\sum_{v} \operatorname{Tr}\right|_{W^{*}[-v]}\left(Q_{W^{*}}(\mu+v) \otimes Q_{V^{*}}\left(\mu+\nu+h^{\left(W^{*}\right)}\right)\right) \circ R_{W^{*} V^{*}}\left(\mu+\nu+h^{\left(V^{*}\right)}+h^{\left(W^{*}\right)}\right) \\
& \circ\left(Q_{W^{*}}\left(\mu+v+h^{\left(V^{*}\right)}\right) \otimes Q_{V^{*}}(\mu+v)\right)^{-1} \circ\left(\operatorname{id}_{W^{*}} \otimes \Psi_{V}(\lambda, \mu+v)\right) \\
= & \left.\sum_{v} \operatorname{Tr}\right|_{W^{*}[v]}\left(\mathbb{Q}_{W^{*}}(\mu+v) \otimes \mathbb{Q}_{V^{*}}\left(\mu+v-h^{\left(W^{*}\right)}\right)\right) \circ \mathbb{R}_{W^{*} V^{*}}\left(\mu+v-h^{\left(V^{*}\right)}-h^{\left(W^{*}\right)}\right) \\
& \circ\left(\mathbb{Q}_{W^{*}}\left(\mu+v-h^{\left(V^{*}\right)}\right) \otimes \mathbb{Q}_{V^{*}}(\mu+v)\right)^{-1} \circ\left(\operatorname{id}_{W^{*}} \otimes \Psi_{V}(\lambda,-\mu-v-\rho)\right) \\
= & \left.\sum_{v} \operatorname{Tr}\right|_{W^{*}[v]}\left(\mathbb{Q}_{W^{*}}(\mu+v) \otimes \mathbb{Q}_{V^{*}}(\mu)\right) \circ \mathbb{R}_{W^{*} V^{*}}(\mu) \circ\left(\mathbb{Q}_{W^{*}}(\mu+v) \otimes \mathbb{Q}_{V^{*}}(\mu+v)\right)^{-1} \\
& \circ\left(\operatorname{id}_{W^{*}} \otimes \Psi_{V}(\lambda,-\mu-v-\rho)\right) \\
= & \left.\mathbb{Q}_{V^{*}}(\mu) \circ \sum_{v} \operatorname{Tr}\right|_{W^{*}[v]} \mathbb{R}_{W^{*} V^{*}}(\mu) \circ\left(\operatorname{id}_{W^{*}} \otimes\left(\mathbb{Q}_{V^{*}}(\mu+v)^{-1} \circ \Psi_{V}(\lambda,-\mu-v-\rho)\right)\right) .
\end{aligned}
$$

Let the Weyl denominator be given by

$$
\delta(\lambda):=e^{\langle\lambda, \rho\rangle} \prod_{\alpha>0}\left(1-e^{-\langle\lambda, \alpha\rangle}\right) .
$$

Define the weighted-trace function by

$$
F_{V}(\lambda, \mu):=\mathbb{Q}_{V^{*}}^{-1}(\mu) \Psi_{V}(\lambda,-\mu-\rho) \delta(\lambda) .
$$

Now replace $W^{*}$ by $W$ in (6.26) and substitute (5.2) and (6.28) in (6.26). We finally obtain the formula which is the $q=1$ case of Theorem 9.2 in [E-S]:

$$
\mathscr{D}_{W}^{\mu, V^{*}} F_{V}(\lambda, \mu)=\chi_{W}\left(e^{-\lambda}\right) F_{V}(\lambda, \mu) .
$$


ACKNOWLEDGEMENTS. I was inspired by Pavel Etingof's lectures on the dynamical Yang-Baxter equations at the London Mathematical Society Symposium on Quantum groups in Durham, UK, July 1999. I thank Eric Opdam for suggesting a shorter proof than I originally had for the rational dependence on $\lambda$ of the intertwining operator.

\section{REFERENCES}

[1] O. Babelon, D. Bernard, and E. Billey, A quasi-Hopf algebra interpretation of quantum 3-j and 6-j symbols and difference equations, Phys. Lett. B 375 (1996), no. 1-4, 89-97, q-alg/9511019. MR 97f:81058. Zbl 894.17008.

[2] P. Etingof and O. Schiffmann, Lectures on the dynamical Yang-Baxter equations, preprint, math.QA/9908064, version 2, 1999, 2000.

[3] P. Etingof and A. Varchenko, Geometry and classification of solutions of the classical dynamical Yang-Baxter equation, Comm. Math. Phys. 192 (1998), no. 1, 77-120, q-alg/9703040. MR 99e:32032. Zbl 915.17018.

[4] Solutions of the quantum dynamical Yang-Baxter equation and dynamical quantum groups, Comm. Math. Phys. 196 (1998), no. 3, 591-640, q-alg/9708015. CMP 1645 196. Zbl 990.35339.

[5] __ Exchange dynamical quantum groups, Comm. Math. Phys. 205 (1999), no. 1, 19-52, math.QA/9801135. CMP 1706 916. Zbl 990.73943.

[6] _ Traces of intertwiners for quantum groups and difference equations, I, math.QA/9907181, version 2, 1999, 2000.

[7] G. Felder, Conformal field theory and integrable systems associated to elliptic curves, Proceedings of the International Congress of Mathematicians, Vol. 1, 2 (Zürich, 1994), Birkhäuser, Basel, 1995, pp. 1247-1255. MR 97m:81015. Zbl 852.17014.

[8] _ Elliptic quantum groups, XIth International Congress of Mathematical Physics (Paris, 1994), Internat. Press, Cambridge, MA, 1995, pp. 211-218, hep-th/9412207. MR 96j:17012.

[9] J.-L. Gervais and A. Neveu, Novel triangle relation and absence of tachyons in Liouville string field theory, Nuclear Phys. B 238 (1984), no. 1, 125-141. MR 86f:81088.

TOM H. KOORNWINDER: KORTEWEG-DE VRIES INSTITUTE, UNIVERSITEIT VAN AMSTERDAM, PlantAge Muidergracht 24, 1018 TV AMSTERDAM, NETHERLANDS

E-mail address: thk@science.uva.n1 


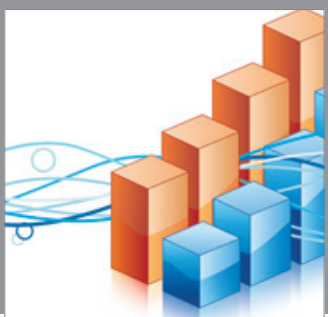

Advances in

Operations Research

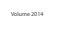

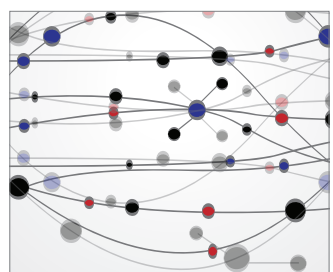

\section{The Scientific} World Journal
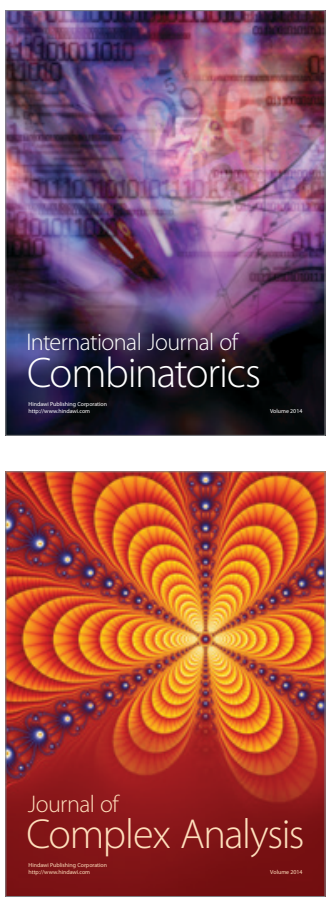

International Journal of

Mathematics and

Mathematical

Sciences
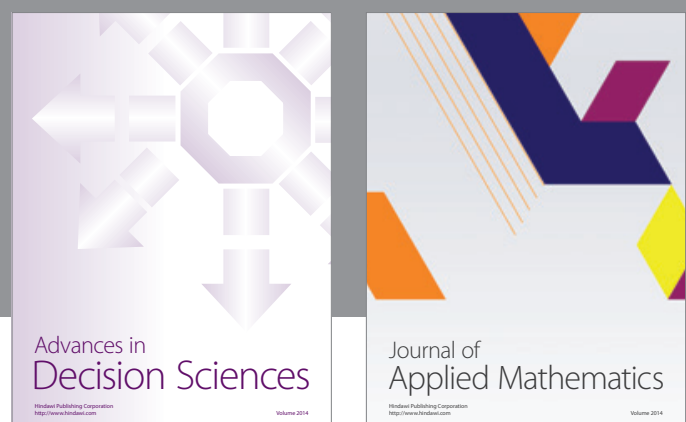

Journal of

Applied Mathematics
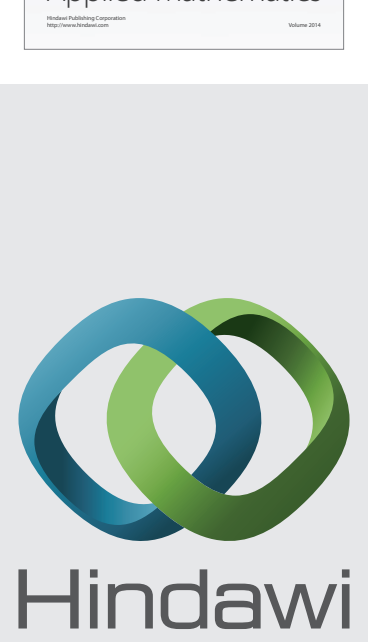

Submit your manuscripts at http://www.hindawi.com
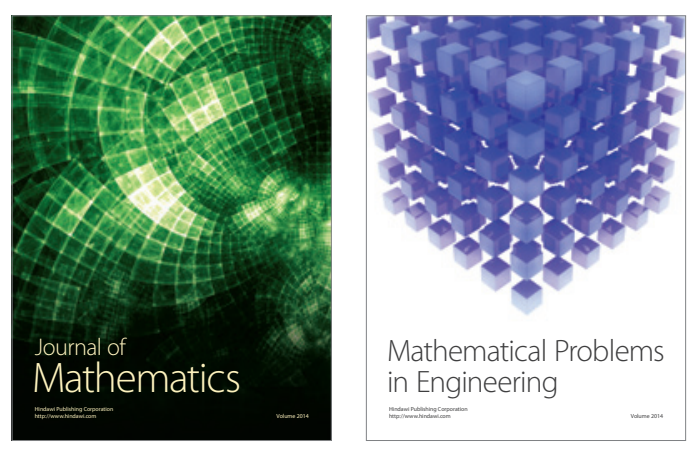

Mathematical Problems in Engineering
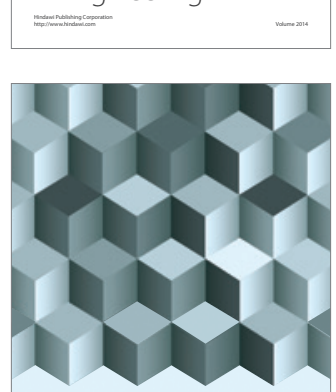

Journal of

Function Spaces
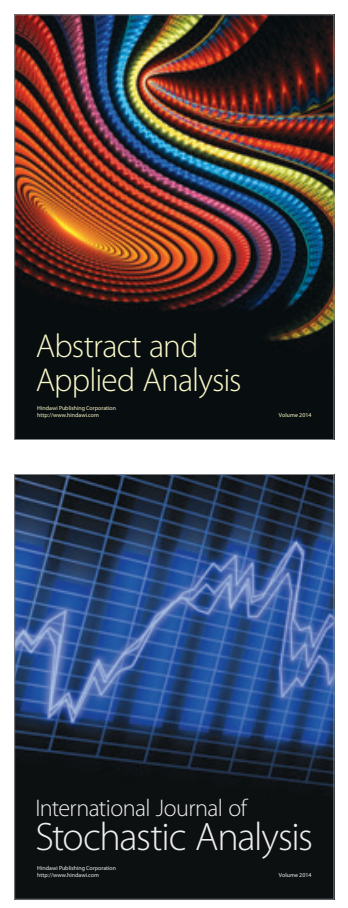

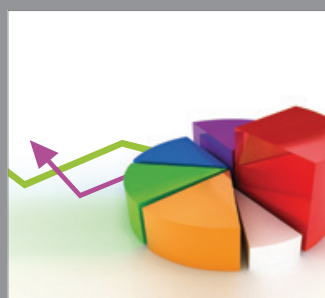

ournal of

Probability and Statistics

Promensencen
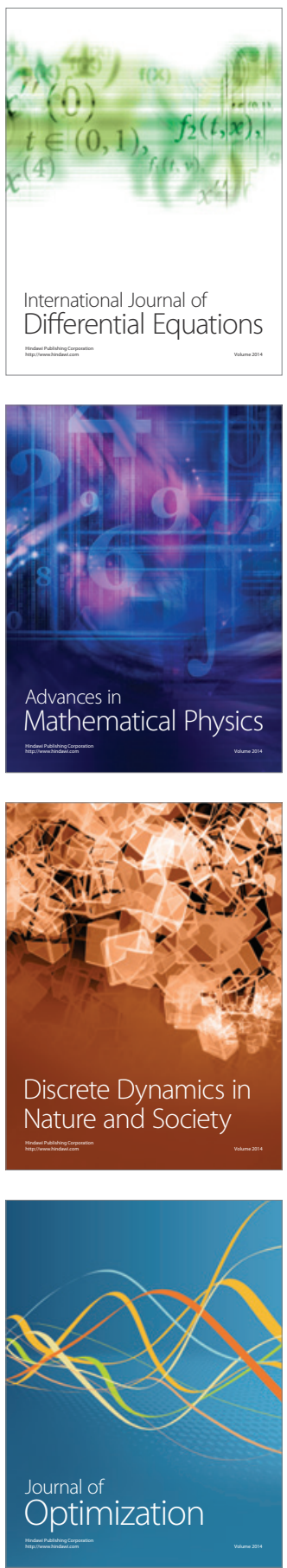